\title{
Don't worry, be active: how to facilitate the detection of errors in immersive virtual environments
}

\author{
Sara Rigutti ${ }^{\text {Corresp., }}{ }^{1}$, Marta Stragà $^{1}$, Marco Jez $^{2}$, Giulio Baldassi ${ }^{1}$, Andrea Carnaghi ${ }^{1}$, Piero Miceu ${ }^{2}$, Carlo \\ Fantoni Corresp. 1 \\ ${ }^{1}$ Department of Life Sciences, Psychology Unit "Gaetano Kanizsa", University of Trieste, Trieste, Italy \\ 2 Area Science Park, Arsenal S.r.L, Trieste, Italy \\ Corresponding Authors: Sara Rigutti, Carlo Fantoni \\ Email address: srigutti@units.it, cfantoni@units.it
}

The current research aims to study the link between the type of vision experienced in a collaborative immersive virtual environment (active vs. multiple passive), the type of error one looks for during a cooperative multi-user exploration of a design project (affordance vs. perceptual violations), and the type of setting in which multi-user perform (field in Experiment 1 vs. laboratory in Experiment 2). The relevance of this link is backed by the lack of conclusive evidence on an active vs. passive vision advantage in cooperative search tasks within software based on immersive virtual reality. Using a yoking paradigm based on the mixed usage of simultaneous active and multiple passive viewings, we found that the likelihood of error detection in a complex 3D environment was characterized by an active vs. multi-passive viewing advantage depending on:

(1) The degree of knowledge dependence of the type of error the passive/active observers were looking for (low for perceptual violations, vs. high for affordance violations), as the advantage tended to manifest itself irrespectively from the setting for affordance, but not for perceptual violations;

(2) The degree of social desirability possibly induced by the setting in which the task was performed, as the advantage occurred irrespectively from the type of error in the laboratory (Experiment 2 ) but not in the field (Experiment 1 ) setting.

Results are relevant to future development of cooperative software based on immersive virtual reality used for supporting the design review. A multi-user design review experience in which designers, engineers and end-users all cooperate actively within the immersive virtual reality wearing their own head mounted display, seems more suitable for the detection of relevant errors than standard systems characterized by a mixed usage of active and passive viewing. 
1 Don't worry, be active: how to facilitate the detection of 2 errors in immersive virtual environments

3

4 Sara Rigutti $^{{ }^{*}}$, Marta Stragà ${ }^{1 *}$, Marco Jez $^{2}$, Giulio Baldassi ${ }^{1}$, Andrea Carnaghi ${ }^{1}$, Piero Miceu $^{2}$, 5 Carlo Fantoni ${ }^{1 *}$

${ }^{1}$ Department of Life Sciences, Psychology Unit "Gaetano Kanizsa," University of Trieste, Trieste, Italy

$6 \quad{ }^{2}$ Arsenal S.r.L., Area Science Park, Trieste, Italy

7

8 *Equally contributing authors

Corresponding Author:

Carlo Fantoni ${ }^{1}$

via Weiss 21, Trieste, 34128, Italy

Email address: cfantoni@units.it 


\section{Abstract}

10 The current research aims to study the link between the type of vision experienced in a

11 collaborative immersive virtual environment (active vs. multiple passive), the type of error one looks for during a cooperative multi-user exploration of a design project (affordance vs. perceptual violations), and the type of setting in which multi-user perform (field in Experiment 1 vs. laboratory in Experiment 2). The relevance of this link is backed by the lack of conclusive evidence on an active vs. passive vision advantage in cooperative search tasks within software based on immersive virtual reality. Using a yoking paradigm based on the mixed usage of simultaneous active and multiple passive viewings, we found that the likelihood of error detection in a complex 3D environment was characterized by an active vs. multi-passive viewing advantage depending on: reality used for supporting the design review. A multi-user design review experience in which

(1) The degree of knowledge dependence of the type of error the passive/active observers were looking for (low for perceptual violations, vs. high for affordance violations), as the advantage tended to manifest itself irrespectively from the setting for affordance, but not for perceptual violations;

(2) The degree of social desirability possibly induced by the setting in which the task was performed, as the advantage occurred irrespectively from the type of error in the laboratory (Experiment 2) but not in the field (Experiment 1) setting.

Results are relevant to future development of cooperative software based on immersive virtual designers, engineers and end-users all cooperate actively within the immersive virtual reality 
wearing their own head mounted display, seems more suitable for the detection of relevant errors than standard systems characterized by a mixed usage of active and passive viewing.

\section{Introduction}

To achieve an efficient visualization of the external world functional to the detection of relevant 3D environmental features, the brain has to integrate retinal information with extraretinal and proprioceptive information about the observer's ego-motion (Wallach, 1987; Braunstein \& Tittle, 1988; Ono \& Steinbach, 1990; Wexler, 2003; Fetsch et al., 2007; Fantoni, Caudek, \& Domini, 2010). Only when the changes in retinal projections are accurately accounted for the sensed ego-motion information, a stable perception of the environment can be established, and environmental features can be efficiently detected. To what extent the brain integrates retinal and extra-retinal information, and whether such integration provides performance advantages in a task which is typical of cooperative multi-user exploration of complex design projects aided by Immersive Virtual Reality (IVR) is still under debate (Jaekl, Jenkin, \& Harris, 2004; Liu, Ward \& Markall, 2007; Teramoto \& Riecke, 2010; Chrastil \& Warren, 2012; Fantoni, Caudek \& Domini, 2014; Bülthoff, Mohler, \& Thornton, 2018). In order to provide an empirical answer to this question, we rely on IVR, and compare active vs. passive seeking performance for different types of design errors. In two experiments we contrasted participants' performance resulting from the dynamic stereoscopic/cyclopean view of a complex 3D environment, during either an active exploration (i.e., active viewing condition) or a passive replay of the exact same optic information, which was self-generated by the active observer (i.e., passive viewing condition). 
51

52

53

54

55

56

57

58

59

60

61

62

63

64

65

66

67

68

69

\subsection{Passive and active observers in standard collaborative IVR systems}

IVR offers a new human-computer interaction paradigm, in which users actively participate in, and interact with a computer-generated environment. The immersive experience offered by technologies, such as cave automatic virtual environment systems or Head Mounted Displays (HMD), leads to a sense of presence in the virtual environment that is experienced to some extent as it were real (e.g., Bowman \& McMahan, 2007). IVR opens up the possibility to reproduce a variety of contexts that in the real world would be risky, costly or unreachable. For this reason, IVR has been applied to a variety of fields, beyond the entertainment industry, like training, simulation, rehabilitation, tele-operation and others (Durlach \& Mavor, 1995; Brooks, 1999; Schell \& Schochet, 2001; Tarr \& Warren, 2002). Traditionally, IVR systems have been developed for single users. The user wears the HMD, interacts within immersive virtual environment and self-generates a 3D dynamic optic information by controlling the viewpoint motion. Viewpoint motion can be obtained by a combination of ego-motion and control devices generally guided by hand (i.e., SpaceMouse, Oculus Touch and Leap Motion). The single user approach has been relevant to support and improve the immersive experience in different contexts, such as design, aesthetics, and 3D objects' visualization (Bayon, Griffiths \& Wilson, 2006; Chen, 2014). However, it disregards the possible co-presence of more users that share the same immersive virtual environment, as in the case of cooperative working activities. The co-presence of the users in immersive virtual environments is typical of design review activities, in architecture, engineering and shipbuilding. In these working environments, a multi-user IVR system is required to support the activity of design review participants, which is mainly based on the collaborative recovery of design errors (Fernández \& Alonso, 2015). Nowadays an 
optimal collaborative IVR system, in which all participants in the design review task wear their own HMD, sharing a common 3D environment through tele-immersion (Tarr \& Warren, 2002), is quite far from being used in real working environments for technological and economical drawbacks (Chen et al., 2015). To overcome this issue, standard collaborative IVR systems are based on a mixed usage of active and passive viewing and supported by HMD and projection screens respectively (e.g., Bayon, Griffiths, \& Wilson, 2006). The visual immersive experience is generated by one observer who wears the HMD (i. e., the project leader of the design review session) and moves his point of view by body movements (encoded by HMD's translations and rotations), and manual control (encoded by different types of devices). His/her virtual experience is paralleled in real-time on a wide screen for collaborators. In this kind of collaborative IVR system, a single participant actively views the scene through self-controlling the viewpoint translation within the immersive virtual environment, while all remaining participants passively observe the scene from an external viewpoint (Bayon, Griffiths, \& Wilson, 2006; Shao, Robotham, \& Hon, 2012). This mixed usage of simultaneous active and multiple passive viewing constitutes the standard system for remote collaboration nowadays, in which users from different geographical locations share virtual environments (Jones, Naef, \& McLundie, 2006; Bassanino et al., 2010; Chen et al., 2015; Fleury et al., 2015; Tanaya et al., 2017). Although this mixed setting provides a sustainable technological solution, it is characterized by a drawback. Indeed, there is now considerable evidence suggesting that active and passive observers, although receiving similar visual input, rely on a different visual experience (Wallach, Stanton \& Becker, 1974; Braunstein \& Tittle, 1988; Wexler, Lamouret \& Droulez, 2001; Fantoni, Caudek \& Domini, 2010; Caudek, Fantoni \& Domini, 2011; Fantoni, 
96 Caudek \& Domini, 2012; Fantoni, Caudek \& Domini, 2014). This difference is known to be due

97 to the dissimilar sensori-motor and cognitive information resulting from an active exploration

98 of the spatial layout relative to the passive observation, see supplemental text in Text S1 for

99 further details (Sherrington, 1906; Ono \& Steinbach, 1990; Wilson et al., 1997; Chance et al.,

100 1998; Christou \& Bülthoff, 1999; Harman, Humphrey \& Goodale, 1999; Wang \& Simons, 1999;

101 Ujike \& Ono, 2001; James et al., 2002; Peh et al., 2002; von Helmholtz, 2002; Wilson \& Péruch,

102 2002; Wexler, 2003; Jaekl, Jenkin, \& Harris, 2004; Naji \& Freeman, 2004; Waller, Loomis \&

103 Haun, 2004; Ono \& Ujike, 2005; Wexler \& van Boxtel, 2005; Colas et al., 2007; Liu, Ward \&

104 Markall, 2007; Waller \& Greenauer, 2007; Fantoni, Caudek \& Domini 2010; Riecke et al., 2010;

105 Teramoto \& Riecke, 2010; Caudek, Fantoni \& Domini, 2011; Meijer \& Van der Lubbe, 2011;

106 Ruddle, Volkova \& Bülthoff, 2011; Ruddle et al., 2011; Chrastil \& Warren, 2012; Fantoni, Caudek

107 \& Domini, 2012; Fantoni, Caudek \& Domini, 2014; Bülthoff, Mohler \& Thornton, 2018). On the

108 basis of these claims, we formulated the main expectation at the basis of our study: the

109 compatibility between retinal and extra-retinal information resulting from ego-motion,

110 together with agency and intentionality, that are at the basis of active but not of passive

111 viewing, which ultimately mimics a standard collaborative IVR environment, should enhance

112 the allocation of attention to relevant features of the 3D spatial layout. We tested this

113 expectation comparing a possible bolstering effect of active vs. passive viewing in collaborative

114 IVR systems based on the mixed usage of simultaneous active and multiple passive viewing. So

115 far, it remains unclear whether this type of advantage occurs in task used in cooperative

116 software based on IVR (Bayon, Griffiths \& Wilson, 2006). 


\section{2. General Method}

118 We addressed our aim by using a task, which is typical in the design review of large scale digital mock-ups, like those employed in ship design (Jones, Naef, and McLundie, 2006; Fernández \&

120 Alonso, 2015): namely a modified hide and seek task (Chandler, Fritz, \& Hala, 1989). In

121 particular, independent groups, which comprised both an active and multiple passive

122 participants, were asked to simultaneously seek for different types of design errors in two

123 different views, namely a dynamic stereoscopic (for the active observer) and dynamic

124 cyclopean (for the passive observers) of a 3D ship corridor. These views either resulted from an

125 active exploration of the IVR (in active viewing condition) or from the passive replay of the

126 exact same optic information self-generated by the active observer (in passive viewing

127 condition). The active observer self-generated the 3D view combining head (through HMD

128 rotations and translations) and hand (through SpaceMouse controller) movements from a

129 sitting position which was similar to the one adopted by passive observers. As the observers

130 performed the task in a multi-user modality, we encoded the number of detected errors at the

131 end of the exploration/observation session (lasting 4 minutes). A series of screenshots (nine in

132 Experiment 1 or ten in Experiment 2, with only eight of them referring to actual design errors

133 and the remaining serving as catch trials) were shown to the group of participants. After each

134 screenshot, participants were asked to raise their hand to indicate whether the screenshot

135 included a design error they saw during the exploration phase.

136 Importantly, our modified hide and seek task, beyond being applicable in both field (i.e., in the

137 form of demonstrative group game during a science and technology exhibit as in Experiment 1),

138 and laboratory settings (i.e., in the form of group experiment as in Experiment 2), involves 
139 relevant visual and cognitive components generally characterizing cooperative technological

140 applications based on IVR. First, it involves visual spatial attention, which is required to perform

141 a visual search of design errors within a complex IVR scene. Visual spatial attention with active

142 exploration of the actively generated/passively observed visual layout regulates the detection

143 of a target feature (i.e., the error) among all potential distracters. Error detection is often

144 involved in cooperative multi-user activity as in the case of design review sessions in

145 architecture, engineering and shipbuilding domains (Fernández \& Alonso, 2015). Secondly, the

146 detection of errors within a complex 3D environment implied that the observer must form a

147 representation of the detected object (i.e., erroneous), retrieve from memory a conventional

148 representation of the object and finally compare the two representations to decide whether

149 the detected object is erroneous or not (MacKay \& James, 2009).

150 As far as we know, no studies, to date have tested the effect of active vs. multi-passive viewing

151 in such a type of task when different types of design errors are involved in the 3D layout. To this

152 purpose, we followed the Norman's categorization of design errors in artifacts (Norman, 1988)

153 and included two different types of design errors in our task with different degree of

154 dependence on knowledge about the environment structure (Fig. 1):

155 (1) Affordance errors in Fig. $1 \mathrm{~A}$ ( $\mathrm{AE}$, high degree), based on affordance violation such as a

156 door handle in the same side of the hinges;

157 (2) Perceptual errors in Fig. 1B (PE, low degree), based on violation of perceptual

158 organization principles like good continuation (such as a misaligned handrail) and colour

159 similarity (such as a blue handrail embedded within a layout of yellow handrail). 
160 According to Norman (1988), the detection of AE is a knowledge-based process. Indeed, the

161 detection of $A E$ results from incongruence between the mental interpretations of artifacts,

162 based on our past knowledge and the experience applied to our perception of an artifact. By

163 contrast, the detection of PE is perceptual based, as it is knowledge independent and results

164 from incongruence directly stemming from the level of perceptual attributes, which are

165 automatically processed, thus not requiring any learning process (i.e., a colour or shape

166 discontinuity in the 3D layout). An AE type of error can thus be detected if the observer

167 possessed knowledge about the conventional structure of the target object (e.g., the observer

168 must know how a door looks like and how it works in order to recognize that the door handle is

169 misplaced). Otherwise, a PE type of error can be detected even in absence of knowledge about

170 target object structure (e.g., the observer does not need to know how the handrail should look

171 like in conventional setting in order to detect the error). Hence, we expected that the likelihood

172 of detecting an error in our task would depend on the type of error (AE Vs. PE) irrespectively

173 from viewing conditions (active or passive). The likelihood of detecting AEs was expected to be

174 smaller than the likelihood of detecting PEs (Expectation 1).

175 We selected the immersive virtual environment from a section of a digital mock-up of a ship, in

176 order to keep our task representative for cooperative software based on IVR. In particular, we

177 choose a 3D ship corridor suitable for real multi-user session of ship design review (Fig. 1D). The

178 structure of the immersive virtual environment was chosen so that the exploration path of the

179 active observers was sequential relative to the ordering of appearance of design errors. This

180 structure assured us that during the hide and seek task different active observers would have: 
181 (1) followed similar pathways; (2) passed at least once through all of the relevant places needed

182 for the potential detection of all errors.

183 In line with the procedures outlined by previous studies, we employed a new adapted version

184 of a yoked design. In yoked design, the passive viewing is often obtained replaying the active exploration of the same scene generated by another participant, or by the same participant

186 (Rogers \& Rogers, 1992; Harman, Humphrey \& Goodale, 1999; James et al., 2002). Differently

187 from this conventional design, our adapted design mimicked the viewing conditions typical of collaborative IVR systems based on the mixed usage of simultaneous active and multiple passive viewings. Therefore in our design, the active exploration and the multiple passive observations took place simultaneously (Meijer \& Van der Lubbe, 2011). The group of passive participants observed on a large screen the dynamic layout generated by the active observer in real-time. This design assures that, within each group, in the active and passive conditions the participants rely on the exact same visual input. This design overcomes the problem of the unbalanced visual exposure of different types of viewers (i.e., active and passive) which is typical of yoked design, in which the participant passively views the optic array that results from the active exploration of a scene, which is generated by another (active) participant (Chrastil \& Warren, 2012). Furthermore, differently from traditional yoked design, in which the passive view is replayed just after the recording of the active exploration (Rogers \& Rogers, 1992; Wexler, Lamouret, \& Droulez, 2001; Wexler, 2003; Fantoni, Caudek, Domini, 2010), our

200 simultaneous active and passive exposition to the dynamic 3D scene prevents us from potential 201 threats to external and internal validity. Indeed, our simultaneous viewing involves a balanced 202 (not unbalanced) temporal ordering of the viewing conditions. 
203 Finally, we purposely unbalanced the amount of sources of information at which the active and

204 passive viewers had access during our task, in order to assess the main aim of our study: to

205 compare the effectiveness of different type of viewing resulting from collaborative IVR systems

206 based on mixed usage of passive and active viewing. Our passive observers (subdivided in small

207 groups) simultaneously viewed the same dynamic scene self-generated from a single active

208 observer (Fig. 2), which was aided by stereoscopic vision (through HMD), extra-retinal and

209 proprioceptive information regarding self-motion derived from head and hand movements, and

210 cognitive control (see supplemental text in Text S1 for further details). We thus expected

211 (Expectation 2) active viewing to be associated with a larger likelihood to detect design errors

212 than passive viewing. This should result in an active vs. multi-passive error detection advantage.

213 Notably, an opposite expectation occurs if the different amount of cognitive load involved in

214 active exploration over passive observation is considered (Expectation 2b). In particular, the

215 active exploration (relative to the passive observation), including demanding and distracting

216 operation to intentionally move and translate the viewpoint, should result in a reduction of the

217 attentional resources needed to perform the search task (Liu, Ward \& Markall, 2007; see 3rd

218 paragraph in Text S1 for further details).

219 We validate the generalizability of the expected active vs. multi-passive advantage by varying the type of setting amongst Experiments, with Experiment 1 involving a field vs. Experiment 2 a

221 laboratory setting. This variation had the purpose of delineating the limits within which the

222 active vs. multi-passive advantage should fall. In particular, field settings (with a reduced

223 control on extraneous variables) are known to induce social desirability biases more than

224 laboratory settings as an instance of the reactivity to the experimental situation (Crowne \& 
Marlowe 1964; Fisher, 1993; Shadish, Cook \& Campbell, 2002). Such reactivity in our task might

226

lead to a ceiling effect given that the most socially desirable response was the one hitting not

227 missing the error.

228 Both Experiments were approved by the Research Ethics Committee of the University of Trieste

229 (approval number 84) in compliance with national legislation, the Ethical Code of the Italian

230 Association of Psychology, and the Code of Ethical Principles for Medical Research Involving

231 Human Subjects of the World Medical Association (Declaration of Helsinki). All participants

232 provided their oral informed consent. The request of oral consent, formulated by the

233 experimenter, made explicit that people not willing to participate in the session should simply

234 not participate or not respond, without any consequence, and emphasis was put on the

anonymous treatment of data which was part of group instructions at the beginning of session.

Written consent (implying identification of every respondent) was redundant, given that the

Experiments were carried out with visitors' groups scheduled according to the program of the

science exhibit within the field setting in Experiment 1 and with classes of psychology courses

within the laboratory setting in Experiment 2. All participants present during the data collection

sessions accepted to respond. The Ethics Committee of the University of Trieste thus approved

241 the informed consent. Dataset is available as (Data S1).

242 2.1. Experiment 1: Hide and seek in a field setting

2.1.1. Participants

Experimental sessions were conducted in a field setting during a science and technology exhibit at Trieste Next 2017 of a custom made IVR system used to support ship design review. Visitors and scholars served as participants according to a well-defined scheduling of the science and 
247 technology exhibit lasting two days (self-selected sampling). All had normal or corrected to

248 normal vision and were naive as to the purpose of the experiment. None of them reported

249 specific visual diseases relevant for our task, like ocular dominance, colour blindness, and

250 severe forms of eyestrain. Ninety-eight participants took part in the Study (Mean age $=14.42$,

$251 S D=2.76$, range $[12,20])$, subdivided in 10 small groups of variable sizes ranging from a

252 minimum number of 5 to a maximum number of 12 . The groups were recruited one after the

253 other during the exhibit. Six groups were composed by middle-school students $(n=61)$, two

254 groups by high-school students $(n=22)$ and two by undergraduate students $(n=15)$. A total of

25510 active participants vs. 88 passive participants were thus recruited, being each group

256 composed by only one active participant. The active observer was selected among those

257 participants who were willing to wear HMD, reported some previous experience with 3D

258 gaming and low levels of motion sickness. This was assessed by orally asking them about

259 previous experience of motion sickness symptoms according to Kennedy et al. (1993):

260 oculomotor (eyestrain, difficulty focusing, blurred vision, and headache), disorientation

261 (dizziness, vertigo), and nausea (stomach awareness, increased salivation and burping).

\section{2.1.2. Apparatus and Stimuli}

263 The active participants' head motions were tracked in real time using an Oculus Rift CV1 Head

264 Mounted Display (with a FOV of $93^{\circ} \times 104^{\circ}$ and a frame rate of $90 \mathrm{fps}$ ) connected with a PC

265 equipped with an Intel Core i7 7700K 4.20 GHz processor with 16GB RAM and nVIDIA GeForce

266 GTX 1080 Ti graphics card. The PC controlled the simultaneous active and passive visualization

267 of our complex immersive virtual environment and sampled the tracker. The immersive virtual

268 environment was rendered through an IVR system designed by Arsenal S.r.L and was updated in 
269 real time according to a combination of head and hand movements of the active observer (Jez

270 et al., 2018). In particular, the translation/rotation of the point of view through the immersive

271 virtual environment was supported by the combination of head movements and manual control

272 through a 3D Connexion SpaceMouse Pro, with 4 Degrees Of Freedom (DOF; i.e., transition in

273 the directions of three directional axes - forward/backward, left/right, up/down - and rotation

274 on the vertical axis). Accelerometers within the HMD together with two camera motion sensors

275 converging with an angle of about $40^{\circ}$ on the active observer's position at rest (at a distance of

276 about $1.5 \mathrm{~m}$ ) were used to calculate the $x, y, z$ coordinates of the observers viewpoint. These

277 coordinates defined the head movements of the active observers and were used by our

278 program to update in real time the geometrical projection of the 3D graphic. A precise

279 visualization of the virtual environment was achieved by carefully calibrating the 3D layout to

280 the height and the inter-ocular distance of each active observer following the Oculus Rift

281 procedure. The virtual reality experience generated by the HMD was paralleled on two large

282 LCD screens (Samsung 50" J5200 Full HD LED set at a screen resolution of $1024 \times 768$ pixels)

283 connected with the PC. The two LCDs simultaneously displayed a cyclopean view of the exact

284 same dynamic optic information generated by the movements of the active observer within the

285 IVR.

286 Figure 1 provides a bird eye view of the virtual environment (Fig. 1D) used for the hide and seek

287 task together with the design errors implemented throughout the path (screenshots, in Fig. 1A

288 and $1 B$, for AEs and PEs, respectively). Our virtual environment consisted of a custom-made

289 digital mock-up of an L-shaped 3D ship corridor. The corridor was $2.8 \mathrm{~m}$ high, $2.4 \mathrm{~m}$ large, and

290 was composed by two straight segments: a first 35 m long segment connecting the starting 
291 position to the corridor's curve (Fig. 1D, horizontal part of the corridor), and a second $40 \mathrm{~m}$

292 segment connecting the corridor's curve to the corridor's end (Fig. 1D, vertical part of the

293 corridor). The corridor included different features, like: floor texture (a green floor with lateral

294 yellow stripes and horizontal arrows indicating the directions), long pipes on the ceiling (with

295 different sizes and colours), doors (five along the wall and one louvered door in the middle of

296 the corridor), security systems (such as fire extinguisher, warning lights), signals (e.g., "fire

297 point", exit), handrails, bollards, and several technical equipment. The screenshots of the eight

298 design errors actually included in the digital mock-up of the corridor (as presented to the

299 participants to the experiment during the response encoding phase) are shown in Fig. $1 \mathrm{~A}$ and

$3001 \mathrm{~B}$, together with the screenshot used as a catch trial (Fig. 1C). The catch trial was a misplaced

301 pipe holder that was not implemented as an actual error in the digital mock-up of the corridor

302 though being visually consistent with the features of the corridor. Within our experimental

303 design, valid screenshots (i.e., those depicting actual errors) were equally subdivided into the

304 two types: AE and PE (Fig. 1A and 1B, respectively).

305

306

307

308

309

310

311

312

313

314

315

316

317

318

319

Figure 1. Screenshots of design errors and the immersive virtual environment used throughout Experiment 1 and Experiment 2. A and B: screenshots of the 8 actual design errors implemented in the digital mock-up of the corridor as presented to our participants during the response encoding phase, subdivided into the two types: affordance violations in A, and perceptual violations in B. Numbers indicate the relative ordering of appearance of violations along the immersive virtual environment explored during the task from the starting position to the corridor's end (in D). C: the two screenshots used as catch trials shown to the participants in an intermixed and randomized order together with the screenshots of the actual design errors used during the response-encoding phase of the experiments. Experiment 1 included the presentation of the only catch trial screenshot 1, Experiment 2 included the presentation of the both catch trials' screenshots ( 1 \& 2). D: a bird eye view of the immersive virtual environment, from the starting position (coded by the blue star) to the corridor's end (orange circles stand for design errors). The immersive virtual environment was the rendering of a digital mock-up of an L-shaped 3D ship corridor along which the 8 design errors were sequentially implemented along the pathway the observer was required to travel (the numbering corresponds to their relative ordering of appearance along the pathway).

AE included (Fig. 1A) a door handle placed too high and in the same side of the hinges (AE1), a

fire extinguisher placed too high (AE2), a door handle placed too low and in the same side of 
320 the hinges (AE3), and a door handle in the same side of the hinges (AE4). PE included (Fig. 1B) a

321 misaligned bollard (PE1), blue handrail embedded within yellow handrails (PE2), a louvered

322 door missing an half (PE3), and a misaligned handrail (PE4). Three independent judges

323 categorized the eight errors into the two categories, with an inter-rater agreement of $94 \%$. By

324 combining our two types of valid screenshots (AE/PE) with the two viewing conditions

325 (active/passive) we obtained four experimental conditions of a mixed factorial design.

326 As shown in Fig. 1D, the design errors depicted within the screenshots were distributed all

327 along the length of the 3D ship corridor, at a distance from the starting point of $16 \mathrm{~m}$ (AE1), 25

$328 m$ (PE1), 31.5 m (AE2), 40 m (PE2), 49.5 m (PE3), 52 m (AE3), 53 m (PE4), 63 m (AE4).

329 The spatial arrangement of the setting is schematized in Fig. 2A, with Fig. 2B showing a

photograph of the actual exposition context taken during a training session on a digital mock-up

different from the one used during the experiment. The active observer comfortably sat on a

stool right in between the two LCD screens, whereas the passive observers were arranged in

front of the LCD screens comfortably sat on stools at a viewing distance of about $100 \mathrm{~cm}$ (Fig.

2A). A $90 \mathrm{~cm}$ wide desk was interposed in between the passive groups of participants and the

screens in order to control at the best the viewing distance (observers were required to sit on

the stools posing the elbows on the margin of the desk). At the viewing distance of $100 \mathrm{~cm}$ the

retinal size subtended by the 3D dynamic layout when presented in full screen was of about

$57.9^{\circ}$ horizontally and $34.5^{\circ}$ vertically.

Figure 2. Experimental setting of Experiment 1. A: schematic view of the experimental setting with three (as constrained by the $90 \mathrm{~cm}$ desk size), and their average viewing distance (100 $\mathrm{cm}$ when sitting with their elbows on the margin of the desk). This distance is taken from the 2 large LCD screens ( $127 \mathrm{~cm}$ diagonal), displaying in real time the exact same 3D, though monoscopic, view the active observer self-generated combining head movements 
346

347

348

349

350

351

352

353

354

355

356

357

are looking at in real time is consistent with PE1. B: a photograph of the setting during the training session with the digital mock-up (a ship thruster) used to familiarize the active observer with the SpaceMouse and the passive observers with the 3D graphic. Notice that the laboratory setting of Experiment 2 reproduced in smaller scale the one shown in the current scheme, including smaller screens though smaller viewing distances in order to equate the two Experiments for the size of the passive displays in term of retinal sizes $\left(57.9^{\circ} \times 34.5^{\circ}\right)$. B photographed by the co-author Carlo Fantoni who gave his permission for publication.

\subsubsection{Procedure}

The experimental procedure included six phases lasting overall 10 minutes.

First, participants were informed about the experimental setting and the instructions of the

modified hide and seek task using a Power Point presentation. The task was described as a

game, in which the best-performing participant would be awarded with a gadget. This

procedure was also aimed to motivate each single participant to perform at his/her best. To

this purpose, an experimenter first explained to the entire group of participants the role they

were about to endorse in the game. Participants were then introduced with their spatial

disposition depending on the group they belonged: passive participants (one in front of the LCD

to the left of the active observer, the other in front of the LCD to the right of the active

observer, as by Fig. 2A) or active participant (right in between the two groups of passive

participants, Fig. 2A). They were then informed about the aim of the game: "to search, find and

memorize for design errors they would have encountered during the exploration of an

immersive virtual environment". Participants were also informed that at the end of the

exploration they would have been administered a brief serial presentation of screenshots. After

each screenshot's presentation they would have been asked to raise their hand if the

screenshot reported an error they saw during the exploration phase of the game.

Second, participants were then assigned to an active $(n=10)$ or passive $(n=88)$ role. 
370 In the third phase, the group of participants was familiarized with the immersive virtual

371 environment they were going to view during the game. During this familiarization phase, all

372 participants watched on the LCD screen a 1-minute video clip showing the same 3D ship

373 corridor they would have been exposed during the exploration phase of the game but without

374 including design errors. Participants were instructed to carefully watch the video clip. In order

375 to encourage subjects to be as accurate as possible in seeking design errors, the experimenter

376 stressed that in the video clip design errors were absent. This information also provided the

377 subjects with a hint on the design errors they would have successively encountered during the 378 experimental phase.

379 Fourth, the active observer was briefly trained in the usage of the SpaceMouse to control the translation of his/her point of view. The training was performed keeping static the HMD and visualizing its view on the LCD screen. A digital mock-up different from the one used during the experimental phase was used for such a phase (see an example in Fig. 2B). The observer

following the instruction of the experimenter was trained for about 1 minute on the main commands of the SpaceMouse (rotation, lateral and back/forth viewpoint translation). During this phase, in order to similarly train the passive observers with the 3D complex structure of the environment, the passive observers were required to look at the same scene the active observer was looking at.

Fifth, the experimental phase took place lasting about 5 minutes ( 1 minutes of instruction +4 minutes of game). Active and passive observers were first required to keep their positions as by instruction (Fig. 2). The experimenter then focused the observers' attention on the 
392

393

394

395

exploration phase of the game without disclosing their identification. Participants were thus explicitly discouraged to use neither verbal (i.e., claims) nor non-verbal behaviour (i.e., gestures) as soon as they detected a design error. The exploration phase of the game then started: the active observer was required to begin moving freely along the 3D ship corridor (now including the eight design errors) in search for design violations. This phase was terminated after 4 minutes of exploration. On average, this duration led to travel along the corridor back and forth for about one time.

Finally, the sixth response-encoding phase took place. Participants were serially presented with nine screenshots: the eight design errors presented serially in the order they appeared along the path-way from the start position to the corridor's end plus the catch trial presented in a random serial position. As by initial instructions, participants were then required to raise their hands after each screenshot if the screenshot reported an error that they found during the 4 minutes exploration phase of the game: the experimenter registered the number of raised hands as well as the participants who raised their hand. At last, participants were thanked, debriefed and awarded with a gadget. Importantly, participants, throughout the game, were never aware of the number and types of design errors included in the 3D ship corridor.

\subsubsection{Results}

We analyzed the individual likelihood of detecting a design error as an index of performance in our modified hide and seek task following Knoblauch \& Maloney (2012) by applying a generalized linear-mixed effect model $(\mathrm{g} / \mathrm{mm})$ with a probit link function to the whole set of individual binary responses on valid screenshots $(1=$ error detected; $0=$ error not detected). In particular, we used a glmm with the type of design error (AE vs. PE) and the viewing condition 
414 (Active vs. Passive) as fixed factors, with by subject and by error (i.e., the eight valid

415 screenshots) random intercepts and by subject random slope for type of design error (Baayen,

416 Davidson \& Bates, 2008). Importantly, this type of analysis has been proved to be optimal for a

417 research in which the distribution of responses and participants across conditions is inevitably

418 unbalanced as in the case of our design in which the proportion of individual responses

419 resulting from the active observers was of about 0.105 . Two-tailed $p$-values were obtained from

420 type 3 F-statistics with the denominator's degrees of freedom calculated by subtracting from

421 the total number of observations the model's degrees of freedom minus one. As indices of

422 effect size we reported the partial eta-square $\left(\eta_{p}^{2}\right)$, and the concordance correlation

423 coefficient, rc. Following Vonesh, Chinchilli \& Pu (1996, but see also Rigutti, Fantoni \& Gerbino,

4242015 ) such a latter index provides a reliable measure, in the -1 to 1 range, of the degree of

425 agreement between observed values and values predicted by generalized linear-mixed effect

426 models. Finally, we reported Cohen's $d$ as a standardized measure of significant difference

427 between means.

428 Disregarding catch trials, the data analysis was based on the $100 \%$ of active participants'

429 individual responses to valid trials ( $n=80$ resulting from the combination of 10 active observers

430 and 8 errors), and $97 \%$ of passive participants' responses ( $n=680$ out of the total of 704

431 responses, resulting from 85 - 3 excluded from the total of 88 - passive observers and 8 errors).

432 We removed three passive observers from the analysis because of the application of two

433 exclusion criteria both aimed at minimizing any possible biasing effect of social desirability

434 intrinsic in our field setting: 
435

436

437

438

439

440

441

442

443

444

445

446

447

448

449

450

451

452

453

454

455

456

(1) relative the sample mean deviation, one passive participant achieved an individual error-detection proportion that deviates from the one of the active observer of the group he/she belonged more than $\pm 2.5 S D$;

(2) two passive participants provided a positive response (i.e., raised their hand) after the catch trial presentation.

According to the result of the a priori power analysis, the total sample size of 760 observations, resulting from the application of the exclusion criteria and entered onto the glmm model in order to estimate the global active vs. multi-passive advantage, resulted to be large enough to rely on reliable statistical conclusions. We performed the power analysis following Faul, Erdfelder, Buchner and Lang (2009, see the section 5.1) and using the generalized linear regression tool of $\mathrm{G}^{*}$ Power (3.1.9.2 version) for testing logistic regression models based on the z-score distributions for the proportion as those implied in our glmm model (with the probit as a link function). In particular, the required sample size resulting from the analysis was equal to 695, which was indeed smaller than our total sample size of 760 observations obtained from the total number of valid binary responses of our 95 participants in our repeated measures design (with 8 trials - 1 valid screenshot each - for each participant). The required sample size was obtained as follows. Based on a pre-experimental planning of the size of our group sessions we estimated an average proportion of active participants within each group of about 0.11 ( 8 passive observers for every single active observer). We entered this ratio as the best estimate of the expected value of our binomial distribution of the binary encoding of the type of viewing condition variable ( $1=$ active; $0=$ passive). We assumed an odds ratio of about 1.86 , resulting from an expected gain in the detection of error proportion of the active over the passive 
457 condition of about 0.15 with $p(\mathrm{HO})=0.5$, a power of 0.80 , and no correlation between our two

458 predictors (i.e., the type of viewing and the type of error). Notably, the required sample size

459 resulted to be still smaller (728) than our total sample size of observations when calculated

460 entering into the analysis (as the average of the binomial distribution associated to our binary

461 set of type of viewings) the actual ratio (0.105) that we observed from the number of active

462 observations (80) and the total number of observations in our design (760).

463 The pattern of average error detection proportion as a function of the type of design error ( $\mathrm{x}$ -

464 axis) and viewing condition (colour coding as by the legend) shown in Fig. 3A is only in part

465 consistent with our expectations. Expectation 2 (not $2 b$ ) indeed holds for AE but not for PE.

Figure 3. Error detection proportion in Experiment 1 (field setting). A: Mean and SE values of error detection proportion in active (green) vs. passive (blue) viewing conditions as a function of the type of error (affordance vs. perceptual) on the abscissa. B-C: Mean and SE values of error detection proportion in active (green) vs. passive (blue) viewing for the four types of affordance errors (in B) and the four types of perceptual errors (in C). The numbers along the abscissa indicate the relative ordering of error's appearance along the exploration path of the 3D ship corridor, from the starting position to the end (same encoding of Fig. 1).

472 This was confirmed by the results of the $\mathrm{g} / \mathrm{mm}$ analysis $(r c=0.52,95 \% \mathrm{Cl}[0.48,0.55])$ revealing an unexpected trend toward significance of the interaction between type of design error and 
$4810.02)$ conditions thus producing a non significant active vs. multi-passive advantage $(-0.03 \pm$

$482 \quad 0.15 ; z=0.43$, one-tailed $p=0.332)$.

483 The $\mathrm{g} / \mathrm{mm}$ analysis further revealed that Expectation 1 was only in part validated by our pattern

484 of data as revealing a main effect of the type of error $\left(F_{1,751}=5.04, p=0.025, \eta_{p}^{2}=0.007,95 \%\right.$

$485 \mathrm{Cl}[0.000,0.020])$ with an overall $\mathrm{g} / \mathrm{mm}$ estimated facilitation for PE over AE of about $0.31 \pm$

4860.17 . However, this facilitation was moderated by the type of viewing, as qualified by the type

487 of viewing $\times$ type of error interaction. The facilitation for PE over AE was indeed present only

488 for passive, but not for active viewing conditions. For the passive viewing condition the

489 likelihood of detecting an error was indeed smaller for AEs $(M=0.53 \pm 0.02)$, rather than PEs

$490(M=0.80 \pm 0.02)$ type of errors $(z=-2.38$, one-tailed $p=0.009, d=0.60)$. No such a difference

491 was found for the active viewing condition in which the likelihood of detecting an error was

492 instead similar for AEs $(M=0.70 \pm 0.07)$ and PEs $(M=0.77 \pm 0.07)$ type of errors $(z=0.47$, one-

493 tailed $p=0.318)$.

494 A further post-hoc $\mathrm{g} / \mathrm{mm}$ analysis was carried out on the distribution of the likelihood of errors

495 detection amongst our two types of errors (Fig. 3B and 3C for affordance and perceptual,

496 respectively). This analysis revealed the active vs. multi-passive advantage for AE type of error

497 was mainly due to two out of the four AEs: AE3 and AE4 (Fig. 3B). We indeed found a significant

498 active vs. multi-passive error detection advantage for AE3 $(z=-2.10$, one-tailed $p=0.018, d=$

$4990.71)$ and AE4 ( $z=-1.84$, one-tailed $p=0.033, d=0.64)$, but not for AE1 $(z=-1.18$, one-tailed $p=$

$5000.120)$ and AE2 $(z=0.92$, one-tailed $p=0.178)$. No reliable differences were instead found for

501 any one of the four PEs (PE1: $z=-0.07$, one-tailed $p=0.470 ;$ PE2: $z=1.24$, one-tailed $p=0.107$;

502 PE3: $z=0.37$, one-tailed $p=0.354 ;$ PE4: $z=-0.21$, one-tailed $p=0.415 ;$ Fig. $3 C$ ). 


\section{2.1.5. Discussion}

504 In the current Experiment, we found a trend toward an active vs. multi-passive viewing

505 advantage with AEs but not PEs. As we hypothesized, the failure to find a difference between

506 active and multi-passive observers in PEs can be the result of an overall ceiling effect. According

507 to Expectation 1, such a ceiling was more likely to occur on PEs rather than AEs, given that PEs

508 was expected to be easier to be detected than AEs. Such a response ceiling is likely to be

509 induced by the type of field setting used in the current experiment favouring positive (hand

510 raised) rather than negative (hand not raised) responses. Positive responses were indeed more

511 socially desirable than negative responses in our task, thus producing a social desirability bias.

512 Given these mixed results, the field experimental setting and the heterogeneous sample that

513 we employed in Experiment 1, we ran a second experiment using a more controlled setting

514 possibly reducing social desirability bias.

\section{2.2. Experiment 2: Hide and seek in a laboratory setting}

516 Would results be similar (as those of Experiment 1) when responses at the basis of error

517 detection are less biased by social factors? In order to answer such a question, we performed

518 Experiment 2 that was a replication of Experiment 1 in a VR laboratory (not a field setting), with

519 a sample of participants more uniform in term of age than the one used in Experiment 1, and

520 with smaller groups of active/passive observers. Furthermore, relative to Experiment 1, in

521 Experiment 2 we further reduced the possible social desirability bias, introducing:

522 (1) a method to validate the truthfulness of the errors reported by the active observers

$523 \quad$ signaled by his/her screenshots; 
524

525

526

527

528

529

530

531

532

533

534

535

536

537

538

(2) an additional catch trial, now reaching a $25 \%$ of catches over the total number of valid trials $(n=8)$;

(3) the removal of the ludic dimension as a context within which the participants were asked to perform the task (i.e., the task was not described as a game and participants were not awarded with gadgets for their performance).

Importantly, the new catch trial was intentionally extracted from an immersive virtual environment apparently different from the one tested during the exploration session. Such an apparent difference should implicitly inform the participants that the series of screenshots presented during the response-encoding phase did not necessarily include actual design errors. This should further reduce the tendency to respond according to social desirability.

\subsubsection{Participants}

One-hundred undergraduates of the University of Trieste (Mean age $=20.60, S D=3.29,[18,40]$ range, $77 \%$ female), all with normal or corrected to normal vision, participated in the experiment in return for course credits. According to our pre-experimental questioning (same as in Experiment 1) our participants did not suffer of any specific visual diseases relevant for our task (ocular dominance, colour blindness, and severe forms of eyestrain). Participants were subdivided in 17 groups with variable size, each of which ranged from three to eight. Groups were tested in individual sessions and were formed following the same procedure used in Experiment 1 . Fourteen groups were composed by first year bachelor psychology students $(n=$ 91), and three groups each composed by three participants included second year master psychology students $(n=9)$. Participants were assigned to active $(n=17)$ and passive $(n=83)$ conditions following the same selection criteria used in Experiment 1. 


\section{2.2.2. Apparatus and Stimuli}

547 The experiment was conducted in a VR laboratory equipped with the same experimental

548 apparatus of Experiment 1, with three major differences:

549 (1) The stereo projection system was driven by an MSI laptop instead of a PC, equipped $100 \mathrm{~cm}$ ) so to equate the displays in term of retinal size.

In order to provide a method that corroborates the reliability of the errors reported by the active observers, the IVR system was aided by a functionality that allows to store in real time the screenshots of the cyclopean views of the active observer. A screenshot was stored during the exploration as soon as the active participant pressed the R button of the Space Mouse.

The spatial disposition of participants within the setting, and the immersive virtual environment explored during the task (the 3D L-shaped ship corridor, Fig. 1D) together with the design errors

(Fig. 1A and 1B) were the same as in Experiment 1. The screenshots used during the responseencoding phase were the same as in Experiment 1 ( 8 valid screenshots, 4 AEs +4 PEs, plus 1 catch trial screenshot), with 1 additional screenshot used as catch trial. This additional screenshot displayed a missing holder in a ship thruster (Fig. 1C, screenshot 2). This catch trial screenshot was apparently different from all of the other screenshots for colour properties, 
568 being reddish not greenish. Such a difference in colour purposely magnifies the un-relatedness

569 of some trials with the 3D ship corridor thus reducing the tendency to favour positive over

570 negative responses in our task. In Experiment 2, the same $2 \times 2$ mixed factorial design of

571 Experiment 1 applied, with four experimental conditions resulting by the full factorial

572 combination of 2 types of design errors $\times 2$ viewing conditions.

\section{2.2.3. Procedure}

574 The procedure was similar to the one used in Experiment 1 with the major difference that the

575 ludic dimension was now intentionally removed from the task so to reduce the social

576 desirability bias. The procedure included the same six phases described in subsection 2.1.3 and

577 lasted almost the same time (10 minutes). Major differences regarded:

578 (1) the first phase, in which task was described as a game, was omitted from the current

$579 \quad$ instruction;

580 (2) in the second phase, in which, although following the same selection criteria used in

581 Experiment 1, albeit a relatively larger number of participants were assigned to the

582 active $(n=17)$ and the passive $(n=83)$ conditions;

583 (3) the fifth experimental phase, that although requiring the participants to perform the 584 same modified hide and seek task of Experiment 1 (lasting 4 minutes), it included an 585 additional task for the active participant (to push the R button of the SpaceMouse as soon as he/she detects a design error during the exploration phase);

(4) the sixth response encoding phase that was conducted following the exact same procedure as in Experiment 1, but that included the serial presentation of 10 (not 9) screenshots. 
590 At last, we collected demographic information (age and gender), and participants were thanked 591 and debriefed following the same procedure used in Experiment 1.

592 The debriefing in Experiment 2 also served the purpose of gathering information from those 593 active observers that during the exploration phase did take a number of screenshots that 594 differs from those signalled by the raising of their hand. Ten observers out of the total of 17 595 took a screenshot of all of the errors they reported during the response-encoding phase. The 596 remaining 7 observers either took no screenshot at all $(n=1)$, or took one $(n=5)$ to three $(n=$ 597 1) screenshot less than the number of errors they reported during the response encoding 598 phase. The average number of correspondence between screenshots and errors reported by 599 the raising of hands was of about $82 \%$. This showed that our response method was reliable. In 600 the debriefing phase, all seven participants with a smaller correspondence between the 601 screenshots they took and the errors they reported claimed that they might have: 1) forgotten

602

603

604 605 606 about pushing the SpaceMouse button during the exploration phase; 2) pushed the SpaceMouse button accidentally or to signal relevant parts of the environment in addition to mere errors. In line with this latest claim, the analysis of the screenshots reveals that observers in general used the SpaceMouse button not only to signal a detection error but also to signal a salient part of the 3D environment. The average individual proportion of screenshots relative to the total number of actual errors $(n=8)$ was indeed reliably larger than the average individual error detection proportion ( $0.72 \pm 0.09$ vs. $0.48 \pm 0.05, t=3.91, d f=16, p=0.001, d=1.95)$. Consequently, we decided not to use the screenshots as an additional exclusion criterion for our participants, given that our result suggested that they were used for a mixture of purposes 
611 (accidental button press, to signal a detection error, or to signal a relevant part of the

612 environment).

\section{2.2.4. Results}

614 As in Experiment 1, we analyzed the individual likelihood of detecting a design error as an index

615 of performance in our modified hide and seek task by applying the same $\mathrm{glmm}$ model (with

616 probit as a link function) to the whole set of individual binary responses on valid screenshots (1

$617=$ error detected; $0=$ error not detected), with type of design error (AE vs. PE) and viewing

618 condition (Active vs. Passive) as fixed factors, and with by subject and by error random

619 intercepts and by subject random slope for type of design error. We used the same indices of

620 effect size as in Experiment 1 in order to support the reliability of our statistical effects.

621 Disregarding catch trials, the data analysis was now based on the $100 \%$ of active participant's

622 responses to valid trials ( $n=136$ resulting from the combination of 17 active observers and 8

623 errors), and on the $96 \%$ of passive participant's responses ( $n=640$ out of the total of 664

624 responses, resulting from 80 - 3 excluded from the total of 83 - passive observers and 8 errors).

625 After the application of the same exclusion criteria used in Experiment 1, we removed from the 626 analysis three passive observers who raised their hand when one of the two catch trials was

627 presented.

628 Performing the same a priori power analysis of Experiment 1 (assuming the same power, and 629 odds ratio), our total sample size of 776 observations entered onto the $\mathrm{g} / \mathrm{mm}$ model resulted to 630 be large enough to rely on reliable statistical conclusions. The sample size was indeed larger

631 than the one used in Experiment 1, and more balanced across viewing conditions, with the 632 planned proportion of active participants within each group raised to 0.2 , which resulted from 
633 planned groups that on average were formed by one active participant out of four passive

634 participants. The increased balance over the two viewing conditions delivered a required

635 sample size of 427 , that was much smaller than the observed one. Notably, the required sample

636 size was still smaller (473) than the one we used also entering into the analysis (as the average

637 of the binomial distribution associated to our binary set of type of viewings) the actual ratio

638 (0.175) that we observed from the number of active observations (136) and the total number of

639 observations in our design.

640 Figure 4A depicts the pattern of average error detection proportion as a function of the type of

641 design error ( $x$-axis) and viewing condition (colour coding as by the legend). The pattern is

642 apparently different from the one of Fig. 3A (Experiment 1), being now consistent with both

643 Expectation 1 (main effect of the type of design error), and Expectation 2 (main effect of

644 viewing condition). Furthermore, differently from Experiment 1 the type of design error $x$

645 viewing condition is now absent.

646 Figure 4. Error detection proportion in Experiment 2 (laboratory setting). A: Mean and SE values of error 647 detection proportion in active (green) vs. passive (blue) viewing conditions as a function of the type of error 648 (affordance vs. perceptual) on the abscissa. B-C: Mean and SE values of error detection proportion in active (green) 649 vs. passive (blue) viewing for the four types of affordance errors (in B) and the four types of perceptual errors (in

$650 \mathrm{C})$. The numbers along the abscissa indicate the relative ordering of error's appearance along the exploration path

651 of the 3D ship corridor, from the start position to the end (same encoding of Fig. 1).

652 These observations were confirmed by the statistical $\mathrm{glmm}$ analyses $(r c=0.58,95 \% \mathrm{Cl}[0.54$,

653 0.61]). Indeed, as consistent with Expectation 2 (not 2b), g/mm results showed a significant

654 main effect of the viewing condition $\left(F_{1,767}=5.02, p=0.025, \eta_{p}^{2}=0.007,95 \% \mathrm{Cl}[0.000,0.019]\right)$,

655 with a larger likelihood of detecting an error in active $(M=0.48 \pm 0.04)$, rather than in passive

656 viewing condition $(M=0.38 \pm 0.02)$. This effect was quantified by an active vs. multi-passive

657 error detection advantage of about $0.15 \pm 0.13(z=2.15$, one-tailed $p=0.016, d=0.20)$. 
658 Expectation 1 was supported by the significant main effect of type of design error $\left(F_{1,767}=8.23\right.$, $\left.659 p=0.004, \eta_{p}^{2}=0.011,95 \% \mathrm{Cl}[0.002,0.026]\right)$, with a larger likelihood of detecting an error for $660 \operatorname{PE}(M=0.56 \pm 0.03)$, rather than for $\mathrm{AE}(M=0.23 \pm 0.02)$. Unlike Experiment 1 , the interaction 661 between type of design error and viewing condition was not statistically significant $\left(F_{1,767}=\right.$ 662 $\left.0.12, p=0.727, \eta_{p}^{2}=0.000,95 \% \mathrm{Cl}[0.000,0.005]\right)$.

As in Experiment 1 we further performed a post-hoc glmm analyses on the way in which the 664 likelihood of errors detection was distributed amongst our two types of errors (Fig. 4B and 4C 665 for affordance and perceptual, respectively). This analysis revealed that the origin of the active vs. multi-passive advantage was likely to be qualified by two out of four AEs (Fig. 4B, AE1, z = 1.52, one-tailed $p=0.064, d=0.38$; and AE2, $z=-1,43$, one-tailed $p=0.077, d=0.38$ ), and two out of four PEs (Fig. 4C, PE1, $z=-1.45$, one-tailed $p=0.073, d=0.37$; and PE3, $z=-1.39$, onetailed $p=0.083, d=0.39)$, all of which showed a trend toward significance concerning the active vs. passive error detection advantage.

\subsubsection{Discussion}

Experiment 2 revealed that both the active vs. multi-passive viewing advantage predicted by

Expectation 2 (not $2 b$ ), and the effect of facilitation due to the type of error predicted by

Expectation 1, did occur under a laboratory setting. Joining data from both Experiments revealed an overall reduction of the tendency to provide a positive response in Experiment 2 relative to Experiment 1 . The likelihood of detecting an error strongly decreased in the laboratory setting of Experiment $2(M=0.40 \pm 0.02)$, relative to the field setting of Experiment 
680 as an additional fixed factor in the $\mathrm{g} / \mathrm{mm}$ analysis. The direction of this effect might be

681 accounted for by social desirability, which more likely biases responses towards positive 682 detection in field rather than in laboratory settings.

\section{3. Conclusions}

684 We reported two experiments on the link between the type of vision one might experience in a 685 collaborative virtual environment (active vs. multiple passive), the type of error one might look 686 for during a cooperative multi-user exploration of a complex design project (affordance vs. 687 perceptual violations), and the type of setting - manipulated through Experiments - within 688 which a multi-user activity is performed (field in Experiment 1 vs. laboratory in Experiment 2). 689 Our two experiments demonstrated that the likelihood of error detection within a complex 3D 690 immersive virtual environment is characterized by an active vs. multi-passive viewing advantage (consistent with our Expectation 2). In particular, we found that such an advantage depends on multiple sources of information, like:

(1) The degree of knowledge dependence of the type of error the passive/active users were looking for (low for perceptual vs. high for affordance violations), as the advantage tended to manifest itself irrespectively from the setting for affordance, but not for perceptual violations. This was suggested by the main effect of the type of viewing in Experiment 2 vs. the type of viewing $\times$ type of error interaction in Experiment 1 . This difference was characterized by an anisotropy: a facilitation in the detection of PEs over AEs (consistent with our Expectation 1) which is particularly evident under the laboratory setting (Experiment 2), given that it also emerges in the active vision conditions. We interpreted such facilitation as a by-product of the relative complexity of 
the encoding process supporting the detection of the error. AEs would involve a more complex encoding process than PEs to be detected, as their encoding depends on previous knowledge about the structure of the object, while PEs being based on mere

Taken together, these results can be reconciled with the somewhat mixed findings stemming

721 from literature on active/passive exploration/observation for error detection and way finding

722 (Liu, Ward \& Markall, 2007; Chrastil \& Warren, 2012; Bülthoff, Mohler \& Thornton, 2018). The 
724 exploration/observation like stereopsis (monoscopic cyclopean view in passive viewers), extra-

725 retinal and proprioceptive information from ego-motion (absent in passive viewers), and

726 agency/intentionality (absent in passive viewers), would have contributed to determinate a

727 superiority of active vs. passive vision in the detection of errors in our complex 3D environment.

728 However, this superiority might result both from the correspondence between retinal and

729 extraretinal egomotion signals, and from the correspondence between retinal and

730 proprioceptive signals from hand movement used to control the viewpoint motion within the

731 immersive virtual environment. This latest information component linked to manual control is

732 indeed known to positively affect perceptual performance in both 3D (Harman, Humphrey \&

733 Goodale, 1999; James et al., 2002) and 2D space (Ichikawa \& Masakura, 2006; Scocchia, et al., 734 2009).

735 Notably, following Liu, Ward \& Markall (2007), a superiority of active over passive vision is only

736 apparently in contrast with the lower cognitive load to which our passive, rather than active,

737 observers were subjected to during our task (with only the active observer being involved

738 during the error detection task also in complex activities required by the active exploration of

739 the environment, like controlling/moving the point of view, deciding where to look for etc.). It

740 was indeed possible that in our task such an active vs. passive disadvantage was overshadowed

741 by an active vs. passive advantage produced by the compatibility between visual and non-visual

742 information, together with agency and intentionality, that characterize active (not passive)

743 vision. These peculiar components might have enhanced the allocation of visual spatial

744 attention towards relevant features of the complex 3D spatial layout we used in our

745 Experiments. According to this interpretation, a perspective point for future studies would be 
746 to test whether this overshadowing could be minimized by reducing the complexity of the 3D

747 environment. This reduction of complexity could lead to a reversed pattern of advantage in line

748 with the one we found in the current study: an active vs. multi-passive disadvantage.

749 In general, our findings may make a useful contribution to the literature on error detection

750 within 3D environments, technology, working sciences and methodology. Our results, indeed

751 allowed us to provide a first tentative response to a relevant though still debated research

752 question: How the effectiveness of the interaction with a complex 3D environment is affected by

753 the different types of devices (HMD vs. screen) mediating the immersive experience offered by

754 VR technologies in collaborative contexts?.

755 In the present study we empirically answered to this question that poses a novel research

756 problem, namely the multi-user vision problem. This problem involves the understanding of

757 whether a self-generated stereoscopic and immersive view of a complex layout leads to a more

758 effective representation of the 3D scene compared to the passive reply of the same optic

759 information simultaneously displayed on flat screens to multiple passive observers. This

760 problem is rooted into actual working collaborative contexts, such as design review sessions in

761 working domains (e.g., architecture, engineering and shipbuilding) in which the co-presence of

762 more users, which share through cooperation the same project, typically occurs. These design

763 review sessions nowadays are supported by standard collaborative IVR systems based on a

764 mixed usage of passive and active viewing combining HMD and projection screens (Bayon,

765 Griffiths \& Wilson, 2006). In general, previous studies have not yet provided conclusive

766 evidence on the superior advantage of a type of viewing over another during 3D interaction in

767 collaborative immersive virtual environments. Active and passive viewing, although being based 
768 on the same visual input, have indeed access to substantially different sources of information

769 (Wexler, Lamouret \& Droulez, 2001; Wexler, 2003; Fantoni, Caudek \& Domini, 2010; Caudek,

770 Fantoni \& Domini, 2011; Fantoni, Caudek \& Domini 2012). Importantly, here we approached for

771 the first time the multi-user vision problem using a novel adaptation of the yoked paradigm

772 (Rogers \& Rogers, 1992; Fantoni, Caudek \& Domini, 2014). Our adapted yoked paradigm

773 reproduced the simultaneous active/multi-passive conditions occurring in standard

774 collaborative IVR systems: observers subdivided into groups, each composed by one active and

775 multiple passive observers, were indeed asked to simultaneously search for and find (a

776 modified hide and seek task) design errors within a complex 3D ship layout. However, although

777 our paradigm mimicked the viewing conditions typical of standard collaborative IVR systems, it

778 was not fully representative of the real interaction conditions characterizing the working

779 environment in which IVR systems are generally used. In our paradigm, indeed active and

780 passive participants were not allowed to interact and communicate each other during the

781 modified hide and seek task. This limit leaves open the question of the generalizability of our

782 active vs. multi-passive viewing advantage to the real working domain, that could be addressed

783 by future research.

784 As a perspective point for the future development of cooperative software based on immersive

785 virtual environments, we believe that our study might provide a relevant hint. A multi-user

786 design review experience in which designers, engineers and end-users, all cooperate actively

787 within the same IVR wearing their own HMD, seems more suitable for the detection of relevant

788 errors, than standard systems characterized by a mixed usage of active and passive viewing.

789 This point is rooted in the pioneering work of Tarr \& Warren (2002), proposing the 
790 development of tele-immersive systems for the support of the design process, in which users

791 do share a common 3D graphical workplace. This idea is particularly relevant for the

792 implementation of future technological solution based on tele-immersive systems for the

793 support of design review that, according to Fernández \& Alonso's (2015) claims, should

794 minimize implementation errors within projects.

795 4. Acknowledgements

796 We would like to thank all participants and Valentina Piccoli for helping with participants during

797 NEXT 2017.

798

799

800

801

802

803

804

805

806

807

808

809

810

\section{References}

Anelli F, Nicoletti R, Borghi AM. 2010. Categorization and action: What about object consistence? Acta Psychologica 133:203-211. DOI: 10.1016/j.actpsy.2009.11.009

Baayen RH, Davidson DJ, Bates DM. 2008. Mixed-effects modeling with crossed random effects for subjects and items. Journal of Memory and Language 59:390-412. DOI: 10.1016/j.jml.2007.12.005

Bassanino M, Wu KC, Yao J, Khosrowshahi F, Fernando T, Skjærbæk J. 2010. The impact of immersive virtual reality on visualisation for a design review in construction. In Proceedings of 2010 14th International Conference Information Visualisation (IV 2010). London: IEEE, 585-589. DOI: 10.1109/IV.2010.85

Bayon V, Griffiths G, Wilson JR. 2006. Multiple decoupled interaction: An interaction design approach for groupware interaction in co-located virtual environments. International Journal of Human-Computer Studies 64:192-206. DOI: 10.1016/j.ijhcs.2005.08.005 
811 Bowman DA, McMahan RP. 2007. Virtual reality: how much immersion is enough?. Computer 40:36-43. DOI: 10.1109/MC.2007.257

813 Braunstein ML, Tittle JS. 1988. The observer-relative velocity field as the basis for effective motion parallax. Journal of Experimental Psychology: Human Perception and Performance 14:582-590. DOI: 10.1037//0096-1523.14.4.582

Brooks F. 1999. What's Real about Virtual Reality? IEEE Computer Graphics \& Applications 19:16-27. DOI: 10.1109/38.799723

Bülthoff I, Mohler BJ, Thornton IM. 2018. Face recognition of full-bodied avatars by active observers in a virtual environment. Vision Research. DOI: 10.1016/j.visres.2017.12.001

820

821

822

823

824

825

826

827

828

829

830

831

832
Caudek C, Fantoni C, Domini F. 2011. Bayesian modeling of perceived surface slant from actively-generated and passively-observed optic flow. PloS One 6:e18731. DOI: 10.1371/journal.pone.0018731

Chance SS, Gaunet F, Beall AC, Loomis JM. 1998. Locomotion mode affects the updating of objects encountered during travel: The contribution of vestibular and proprioceptive inputs to path integration. Presence: Teleoperators and Virtual Environments 7:168-178. DOI: $10.1162 / 105474698565659$

Chandler M, Fritz AS, Hala S. 1989. Small-scale deceit: Deception as a marker of two-, three-, and four-year-olds' early theories of mind. Child development 60:1263-1277. DOI: $10.2307 / 1130919$

Chen H, Lee AS, Swift M, Tang JC. 2015. 3D collaboration method over HoloLens ${ }^{\mathrm{TM}}$ and Skype ${ }^{\mathrm{TM}}$ end points. In Proceedings of the 3rd International Workshop on Immersive Media Experiences. Brisbane: ACM, 27-30. DOI: 10.1145/2814347.2814350 
833 Chen N. 2014. Inside leap motion: 5 hands-on tips for developing in VR. Leap motion [blog].

834 Available at: http://blog.leapmotion.com/inside-leap-motion-5-hands-on-tips-for-

835 developing-in-virtual-reality/ (Accessed 23 February 2018)

836 Chrastil ER, Warren, WH. 2012. Active and passive contributions to spatial

837 learning. Psychonomic Bulletin Review 19:1-23. DOI: 10.3758/s13423-011-0182-x

838 Christou CG, Bülthoff HH. 1999. View dependence in scene recognition after active learning.

$839 \quad$ Memory \& Cognition 27:996-1007. DOI: 10.3758/BF03201230

840 Colas F, Droulez J, Wexler M, Bessiere P. 2007. A unified probabilistic model of the perception

841 of three-dimensional structure from optic flow. Biological Cybernetics 97:461-477. DOI:

842 $10.1007 / \mathrm{s} 00422-007-0183-z$

843 Costantini M, Ambrosini E, Scorolli C., Borghi AM. 2011. When objects are close to me:

844 Affordances in the peripersonal space. Psychonomic Bulletin \& Review 18:302-308. DOI: $10.3758 / s 13423-011-0054-4$

Crowne D, Marlowe D. 1964. The approval motive. New York: Wiley.

847 Durlach N, Mavor A. 1995. Virtual Reality: Scientific and Technological Challenges, Washington, DC: National Academy Press. DOI: 10.17226/4761

Fantoni C, Caudek C, Domini F. 2010. Systematic distortions of perceived planar surface motion in active vision. Journal of Vision 10:12. DOI: 10.1167/10.5.12

851 Fantoni C, Caudek C, Domini F. 2012. Perceived surface slant is systematically biased in the actively-generated optic flow. PloS One 7:e33911. DOI: 10.1371/journal.pone.0033911

853 Fantoni C, Caudek C, Domini F. 2014. Misperception of rigidity from actively generated optic flow. Journal of Vision 14:10. DOI: 10.1167/14.3.10 
855 Fantoni C, Rigutti S, Piccoli V, Sommacal E, Carnaghi A. 2016. Faster but less careful prehension

856 in presence of high, rather than low, social status attendees. PloS One 11:e0158095.

857 DOI: 10.1371/journal.pone.0158095

858 Faul F, Erdfelder E, Buchner A, Lang AG. 2009. Statistical power analyses using G* Power 3.1:

859 Tests for correlation and regression analyses. Behavior research methods 4: 1149-1160.

$860 \quad$ DOI: $10.3758 / B R M .41 .4 .1149$

861 Fernández RP, Alonso V. 2015. Virtual Reality in a shipbuilding environment. Advances in

862 Engineering Software 81:30-40. DOI: 10.1016/j.advengsoft.2014.11.001

863 Fetsch CR, Wang S, Gu Y, DeAngelis GC, Angelaki DE. 2007. Spatial reference frames of visual,

864 vestibular, and multimodal heading signals in the dorsal subdivision of the medial

865 superior temporal area. Journal of Neuroscience, 27: 700-712.

866 Fisher RJ. 1993. Social desirability bias and the validity of indirect questioning. Journal of consumer research 20:303-315. DOI: 10.1086/209351

868 Fleury C, Ferey N, Vézien JM, Bourdot P. 2015. Remote collaboration across heterogeneous

869 large interactive spaces. In 2015 IEEE Second VR International Workshop on Collaborative Virtual Environments (3DCVE). Arles: IEEE, 9-10. DOI:

871 10.1109/3DCVE.2015.7153591

872 Gerlach C. 2009. Category-specificity in visual object recognition. Cognition 111:281-301. DOI: 10.1016/j.cognition.2009.02.005

874 Harman KL, Humphrey GK, Goodale MA. 1999. Active manual control of object views facilitates 875 visual recognition. Current Biology 9:1315-1318. DOI: 10.1016/S0960-9822(00)80053-6 
876 Jaekl PM, Jenkin MR, Harris LR. 2005. Perceiving a stable world during active rotational and 877 translational head movements. Experimental Brain Research 163:388-399. DOI: $10.1007 / \mathrm{s} 00221-004-2191-8$

879 James KH, Humphrey GK, Vilis T, Corrie B, Baddour R, Goodale MA. 2002. "Active" and "passive" 880 learning of three-dimensional object structure within an immersive virtual reality 881 environment. Behavior Research Methods, Instruments, \& Computers 34:383-390. DOI:

$882 \quad 10.3758 / B F 03195466$

883 Jez M, Fantoni C, Keber M, Rigutti S, Stragà M, Miceu P, Ambrosio L, Carnaghi A, Gerbino W. 884 2018. A Shared Immersive Virtual Environment for Improving Ship Design Review. 885 Technology and Science for the Ships of the Future: 770 - 777. DOI: 10.3233/978-161499-870-9-770.

887

Jones BS, Naef M, McLundie M. 2006. Interactive 3D environments for ship design review and 888 simulation. In Proceedings of the 5th International Conference on Computer Applications and Information Technology in the Maritime Industries. Oegstgeest: IMarEST Benelux

890 Branch, 409-418.

891

Kennedy RS, Lane, NE, Berbaum KS, \& Lilienthal MG. 1993. Simulator sickness questionnaire: An enhanced method for quantifying simulator sickness. The InternationalJournal of Aviation Psychology 3:203-220. DOI: 10.1207/s15327108ijap0303_3

Knoblauch K, Maloney LT. 2012. Modeling psychophysical data in R (Vol. 32). New York: Springer Science \& Business Media.

896 Ichikawa M, Masakura Y. 2006. Manual control of the visual stimulus reduces the flash-lag 897 effect. Vision Research 46: 2192-2203. 
898 Liu CH, Ward J, Markall H. 2007. The role of active exploration of 3D face stimuli on recognition

899

900

901

902

903

904

905

906

907

908

909

910

911

912

913

914

915

916

917

918

919 memory of facial information. Journal of Experimental Psychology: Human Perception and Performance 33:895-904. DOI: 10.1037/0096-1523.33.4.895

MacKay DG, James, LE. 2009. Visual cognition in amnesic HM: selective deficits on the What'sWrong-Here and Hidden-Figure tasks. Journal of Clinical and Experimental Neuropsychology 31:769-789. DOI: 10.1080/13803390802502606

Meijer F, Van der Lubbe, RH. 2011. Active exploration improves perceptual sensitivity for virtual 3D objects in visual recognition tasks. Vision Research 51:2431-2439. DOI: 10.1016/j.visres.2011.09.013

Naji JJ, Freeman TC. 2004. Perceiving depth order during pursuit eye movement. Vision Research 44:3025-3034. DOI: 10.1016/j.visres.2004.07.007

Norman DA. 1988. The psychology of everyday things. New York: Basic Books.Ono H, Steinbach MJ. 1990. Monocular without stereopsis with and head movement. Perception \& Psychophysics 48:179-187. DOI: 10.3758/BF03207085

Ono H, Ujike H. 2005. Motion parallax driven by head movements: Conditions for visual stability, perceived depth, and perceived concomitant motion. Perception 34:477-490. DOI: $10.1068 / p 5221$

Peh CH, Panerai F, Droulez J, Cornilleau-Pérès V, Cheong LF. 2002. Absolute distance perception during in-depth head movement: Calibrating optic flow with extra-retinal information. Vision Research 42:1991-2003. DOI: 10.1016/S0042-6989(02)00120-7

Riecke BE, Bodenheimer B, McNamara TP, Williams B, Peng P, Feuereissen D. 2010. Do we need to walk for effective virtual reality navigation? Physical rotations alone may suffice. In 
920

921

922

923

924

925

926

927

928

929

930

931

932

933

934

935

936

Holscher C, Shipley T, Olivetti Belardinelli M, Bateman J, Newcombe N, eds. Spatial cognition VII: Lecture notes in computer science. Berlin: Springer, 234-247. DOI: 10.1007/978-3-642-14749-4_21

Rigutti S, Fantoni C, Gerbino W. 2015. Web party effect: a cocktail party effect in the web environment. PeerJ 3:e828. DOI: 10.7717/peerj.828

Rogers S, Rogers BJ. 1992. Visual and nonvisual information disambiguate surfaces specified by motion parallax. Perception \& Psychophysics 52:446-452. DOI: 10.3758/BF03206704

Ruddle RA, Volkova E, Bülthoff HH. 2011. Walking improves your cognitive map in environments that are large-scale and large in extent. ACM Transactions on ComputerHuman Interaction, 18 [Article No. 10]:1-20. DOI: 10.1145/1970378.1970384

Ruddle RA, Volkova E, Mohler B, Bülthoff HH. 2011. The effect of landmark and body-based sensory information on route knowledge. Memory \& Cognition 39:686-699. DOI: $10.3758 / s 13421-010-0054-z$

Schell J, Shochet J. 2001. Designing Interactive Theme Park Rides. IEEE Computer Graphics \& Applications 21:11-13. DOI: 10.1109/38.933519

Scocchia L, Grosso RA, de'Sperati C, Stucchi N, Baud-Bovy G. 2009. Observer's control of the moving stimulus increases the flash-lag effect. Vision research, 49: 2363-2370.

Shadish W, Cook TD, Campbell DT. 2002. Experimental and quasi-experimental designs for generalized causal inference. Boston: Houghton Mifflin.

Shao F, Robotham AJ, Hon KK. 2012. Development of a 1: 1 Scale True Perception Virtual Reality System for design review in automotive industry. In Advances in Manufacturing 
941

942

943 Sherrington C. 1906. The Integrative Action of the Nervous System. New York: Charles Scribners $944 \quad$ Sons.

94

946

947

948

949

950

951

952

953

954

955

956

957

958

959

960

961

Technology - XXVI: Proceedings of the 10th International Conference on Manufacturing Research (ICMR2012) 2:468-473.

Tanaya M, Yang K, Christensen T, Li S, O'Keefe M, Fridley J, Sung K. 2017. A Framework for analyzing AR/VR Collaborations: An initial result. In 2017 IEEE International Conference on Computational Intelligence and Virtual Environments for Measurement Systems and Applications (CIVEMSA) Annecy: IEEE, 111-116. DOI: 10.1109/CIVEMSA.2017.7995311

Tarr MJ, Warren WH. 2002. Virtual reality in behavioral neuroscience and beyond. Nature Neuroscience 5:1089-1092. DOI: 10.1038/nn948

Teramoto W, Riecke BE. 2010. Dynamic visual information facilitates object recognition from novel viewpoints. Journal of Vision 10:11. DOI: 10.1167/10.13.11

Ujike H, Ono H. 2001. Depth thresholds of motion parallax as a function of head movement velocity. Vision Research 41:2835-2843. DOI: 10.1016/S0042-6989(01)00164-X

Vonesh EF, Chinchilli VM, Pu K. 1996. Goodness-of-fit in generalized nonlinear mixed-effects models. Biometrics 52(2):572-587. DOI 10.2307/2532896

von Helmholtz H. 2002. Handbook of physiological optics. New York: Dover.

Wallach H. 1987. Perceiving a stable environment when one moves. Annual review of psychology 38:1-29. DOI: 10.1146/annurev.ps.38.020187.000245

Wallach H, Stanton L, Becker D. 1974. The compensation for movement-produced changes in object orientation. Perception \& Psychophysics 15:339-343. DOI: 10.3758/BF03213955 
962 Waller D, Greenauer N. 2007. The role of body-based sensory information in the acquisition of 963 enduring spatial representations. Psychological Research 71:322-332. DOI:

964

965

966

967

968

969

970

971

972

973

974

975

976

977

978

979

980

981 10.1007/s00426-006-0087-x

Waller D, Loomis JM, Haun DB. 2004. Body-based senses enhance knowledge of directions in large-scale environments. Psychonomic Bulletin \& Review 11:157-163. DOI:

\subsection{8/BF03206476}

Wang RF, Simons DJ. 1999. Active and passive scene recognition across views. Cognition 70:191-210. DOI: 10.1016/S0010-0277(99)00012-8

Wexler M. 2003. Voluntary head movement and allocentric perception of space. Psychological Science 14:340-346. DOI: 10.1111/1467-9280.14491

Wexler M, Lamouret I, Droulez J. 2001. The stationarity hypothesis: An allocentric criterion in visual perception. Vision Research 41:3023-3037. DOI: 10.1016/S0042-6989(01)00190-0

Wexler M, van Boxtel JJ. 2005. Depth perception by the active observer. Trends in Cognitive Sciences 9:431-438. DOI: 10.1016/j.tics.2005.06.018

Wilson PN, Foreman N, Gillett R, Stanton D. 1997. Active versus passive processing of spatial information in a computer-simulated environment. Ecological Psychology 9:207-222. DOI: 10.1207/s15326969eco0903_3

Wilson PN, Péruch P. 2002. The influence of interactivity and attention on spatial learning in a desk-top virtual environment. Cahiers de Psychologie Cognitive/Current Psychology of Cognition 21:601-633. 


\section{Figure 1}

Screenshots of design errors and the immersive virtual environment used throughout Experiment 1 and Experiment 2.

A and B: screenshots of the 8 actual design errors implemented in the digital mock-up of the corridor as presented to our participants during the response encoding phase, subdivided into the two types: affordance violations in $\mathrm{A}$, and perceptual violations in $\mathrm{B}$. Numbers indicate the relative ordering of appearance of violations along the immersive virtual environment explored during the task from the starting position to the corridor's end (in D). C: the two screenshots used as catch trials shown to the participants in an intermixed and randomized order together with the screenshots of the actual design errors used during the response-encoding phase of the experiments. Experiment 1 included the presentation of the only catch trial screenshot 1, Experiment 2 included the presentation of the both catch trials' screenshots $(1 \& 2)$. D: a bird eye view of the immersive virtual environment, from the starting position (coded by the blue star) to the corridor's end (orange circles stand for design errors). The immersive virtual environment was the rendering of a digital mock-up of an L-shaped 3D ship corridor along which the 8 design errors were sequentially implemented along the pathway the observer was required to travel (the numbering corresponds to their relative ordering of appearance along the pathway). 

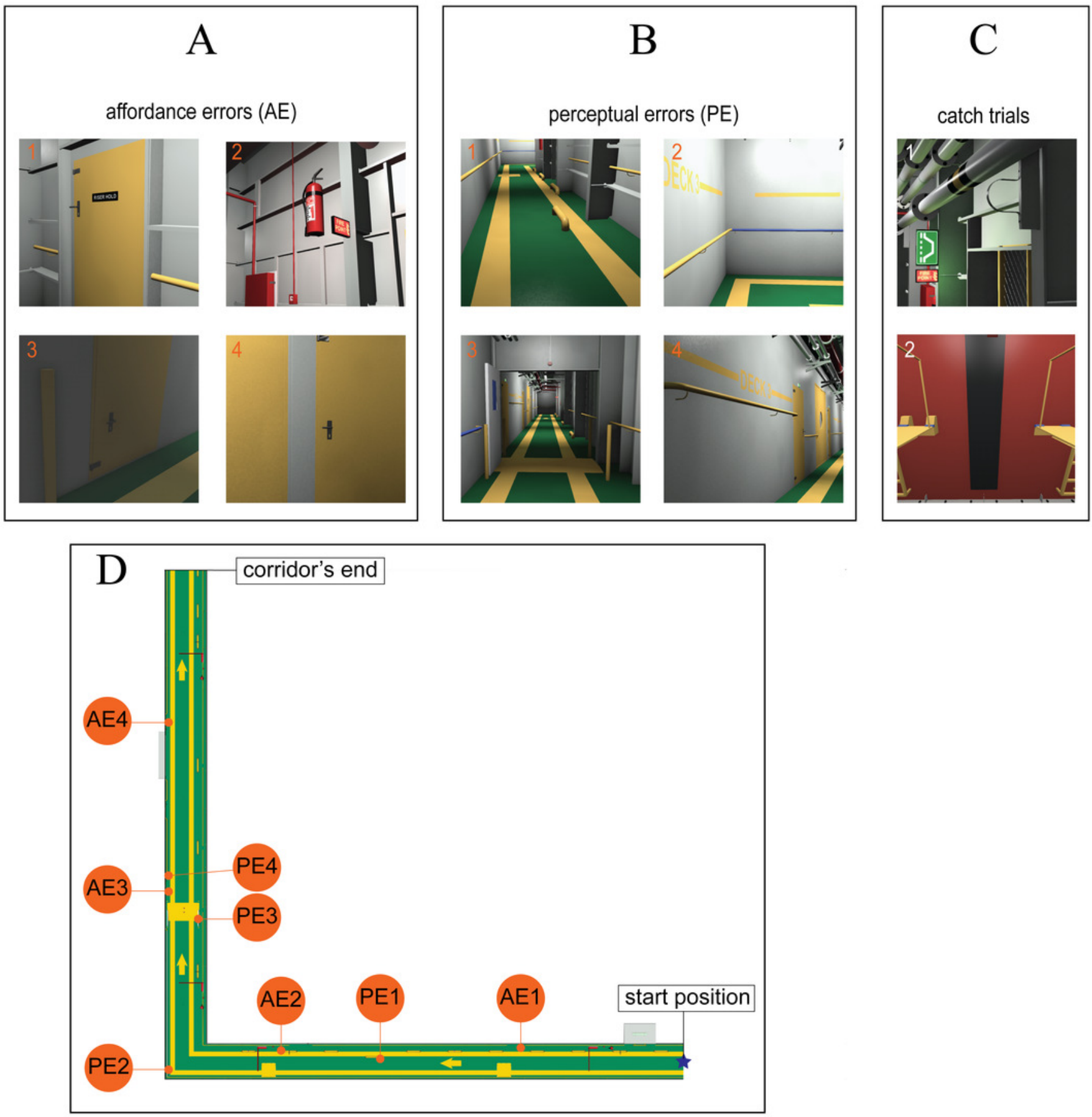


\section{Figure 2}

Experimental setting of Experiment 1.

A: schematic view of the experimental setting with three observers (one active, green arrow, and two passives, blue arrows) implemented in the field setting during the science and technology exhibit of Experiment 1, with superposed the minimal distance the passive observers stand (as constrained by the $90 \mathrm{~cm}$ desk size), and their average viewing distance (100 cm when sitting with their elbows on the margin of the desk). This distance is taken from the 2 large LCD screens ( $127 \mathrm{~cm}$ diagonal), displaying in real time the exact same 3D, though monoscopic, view the active observer self-generated combining head movements and SpaceMouse control. In this example, the view produced by the active observer that the 3 passive observers are looking at in real time is consistent with PE1. B: a photograph of the setting during the training session with the digital mock-up (a ship thruster) used to familiarize the active observer with the SpaceMouse and the passive observers with the 3D graphic. Notice that the laboratory setting of Experiment 2 reproduced in smaller scale the one shown in the current scheme, including smaller screens though smaller viewing distances in order to equate the two Experiments for the size of the passive displays in term of retinal sizes $\left(57.9^{\circ} \times 34.5^{\circ}\right)$. B photographed by the co-author Carlo Fantoni who gave his permission for publication. 


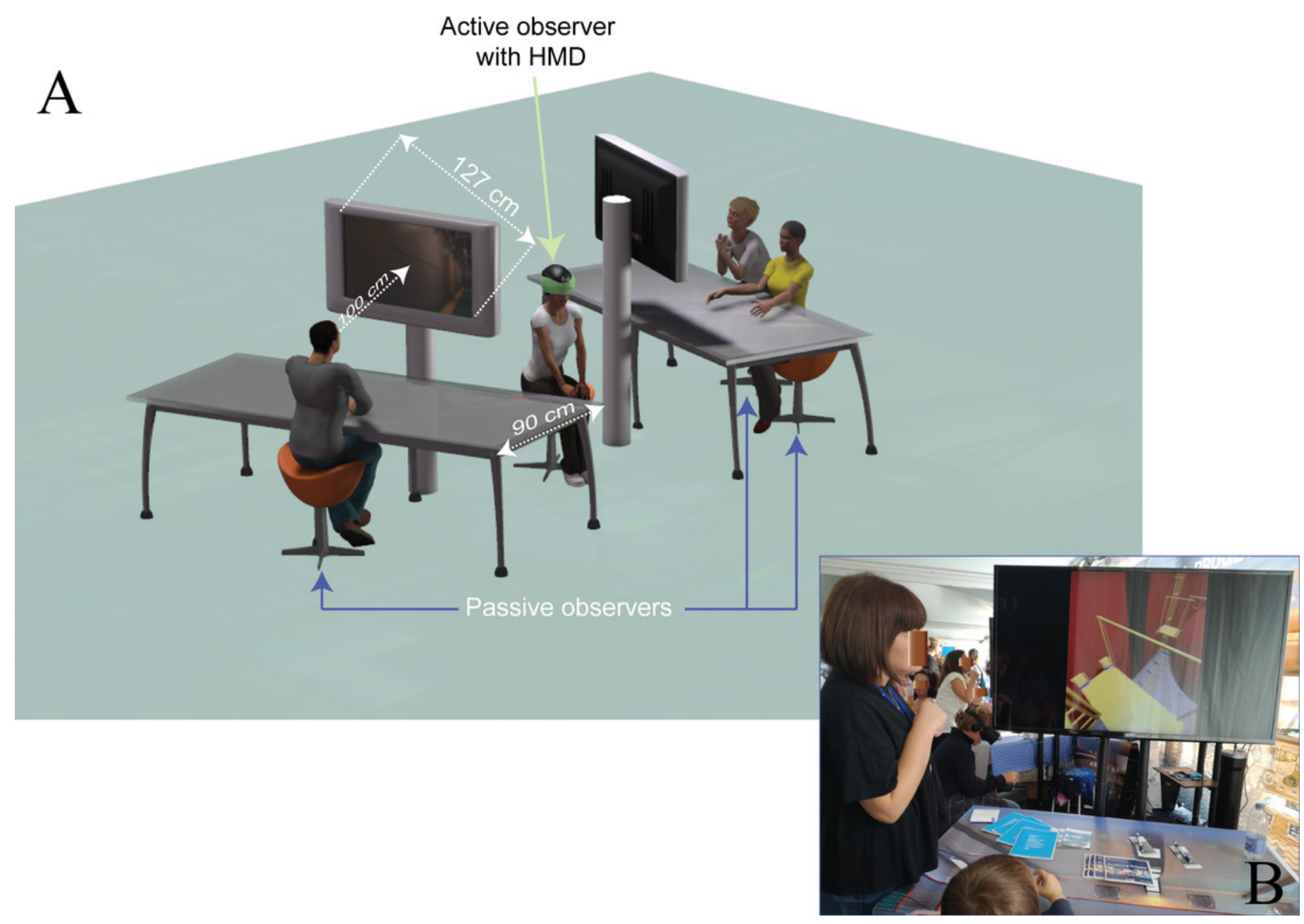


Figure 3 (on next page)

Error detection proportion in Experiment 1 (field setting).

A: Mean and SE values of error detection proportion in active (green) vs. passive (blue) viewing conditions as a function of the type of error (affordance vs. perceptual) on the abscissa. B-C: Mean and SE values of error detection proportion in active (green) vs. passive (blue) viewing for the four types of affordance errors (in B) and the four types of perceptual errors (in C). The numbers along the abscissa indicate the relative ordering of error's appearance along the exploration path of the 3D ship corridor, from the starting position to the end (same encoding of Fig. 1). 

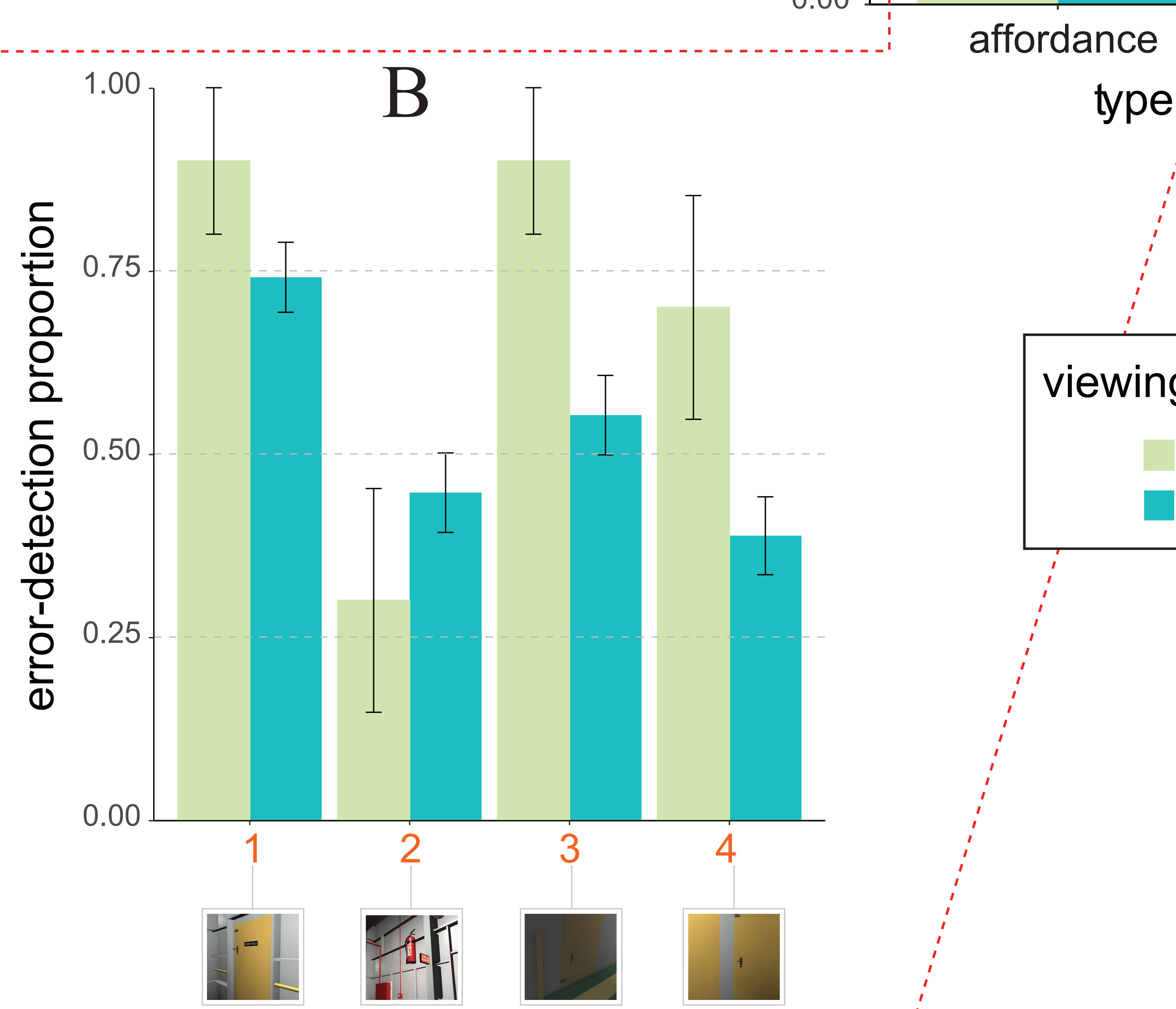

perceptual

0.00

affordance
type of error

affordance errors $(A E)$

viewing condition

active

passive

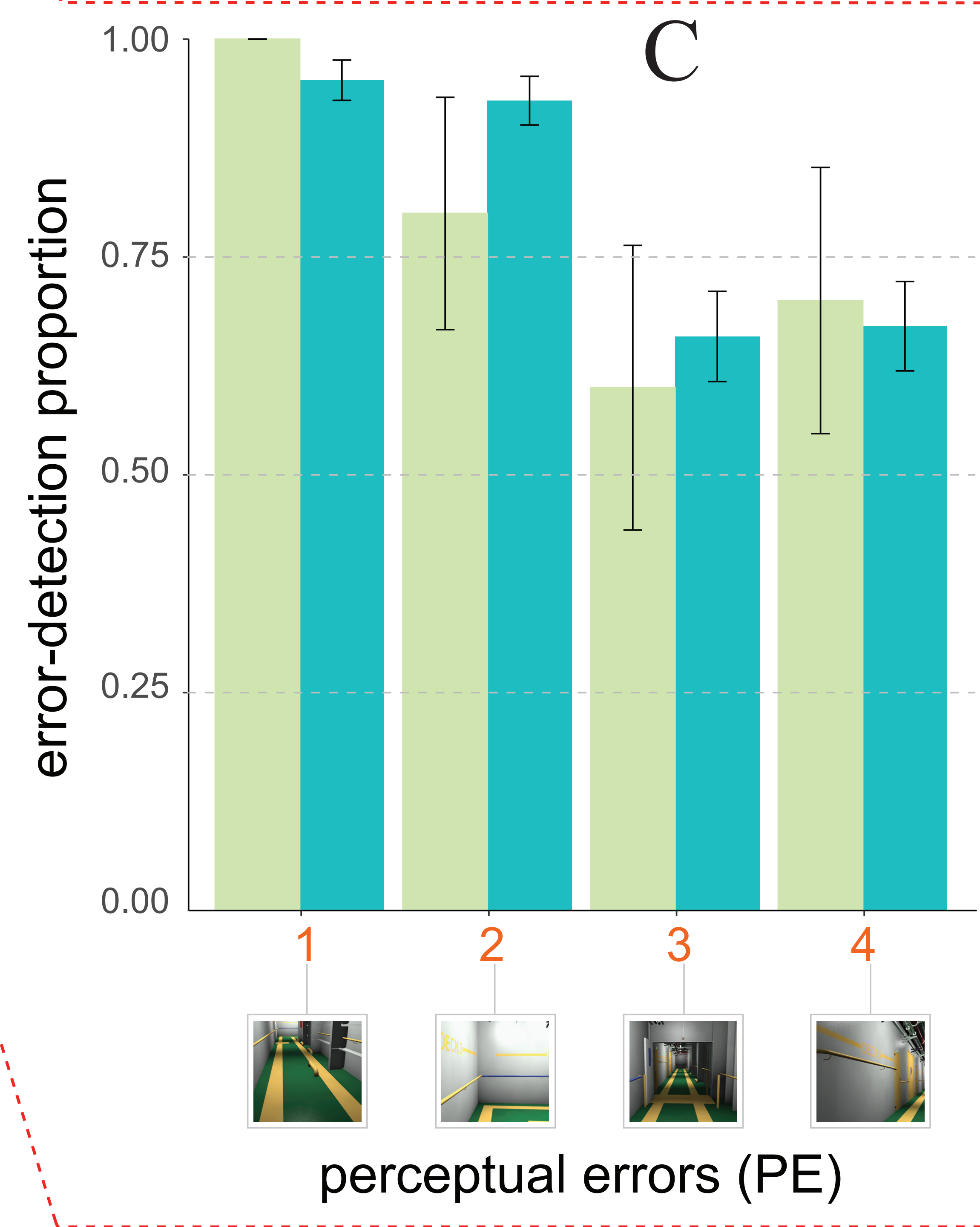


Figure 4 (on next page)

Error detection proportion in Experiment 2 (laboratory setting).

A: Mean and SE values of error detection proportion in active (green) vs. passive (blue) viewing conditions as a function of the type of error (affordance vs. perceptual) on the abscissa. B-C: Mean and SE values of error detection proportion in active (green) vs. passive (blue) viewing for the four types of affordance errors (in B) and the four types of perceptual errors (in C). The numbers along the abscissa indicate the relative ordering of error's appearance along the exploration path of the 3D ship corridor, from the start position to the end (same encoding of Fig. 1). 


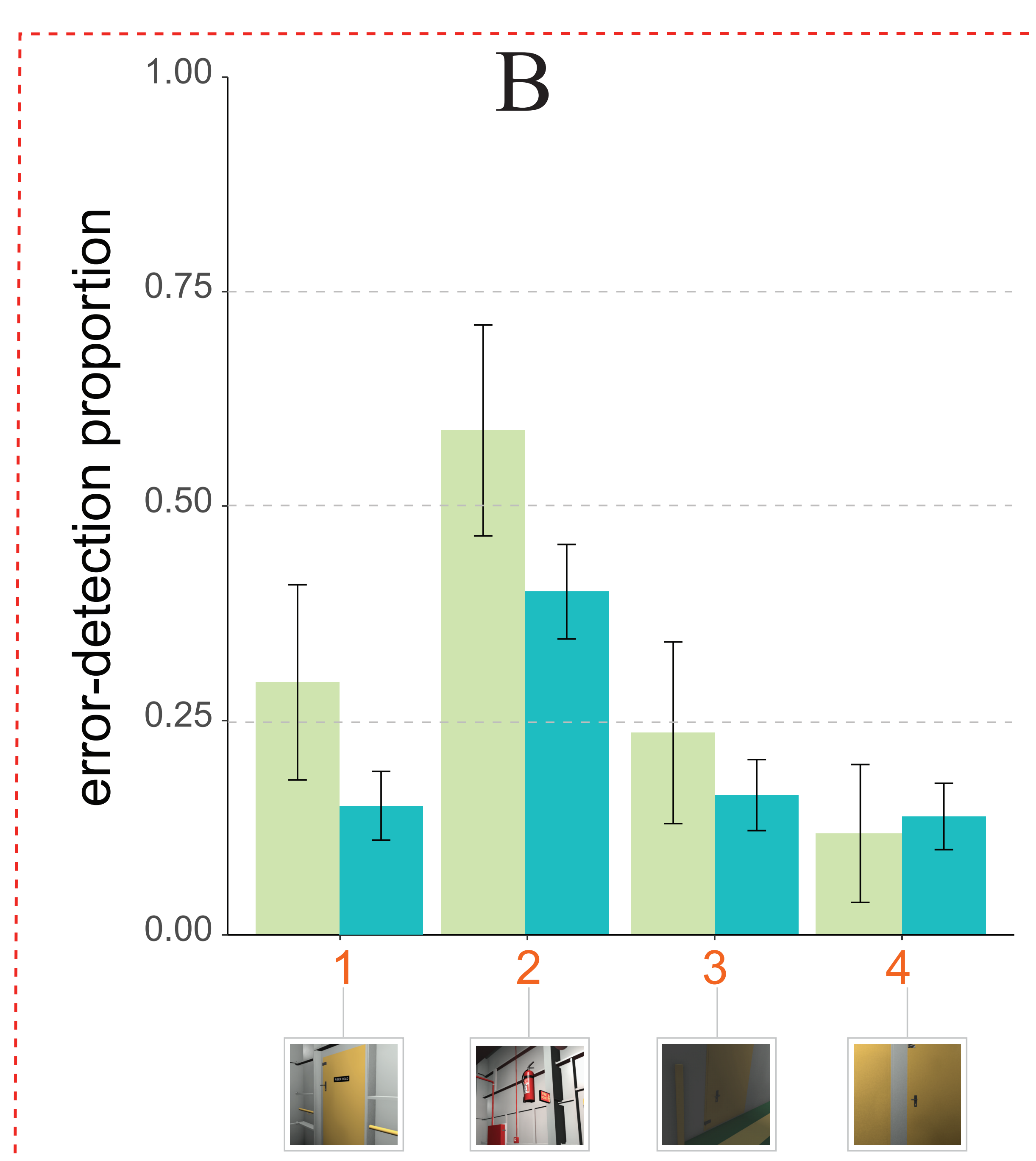

affordance errors (AE)

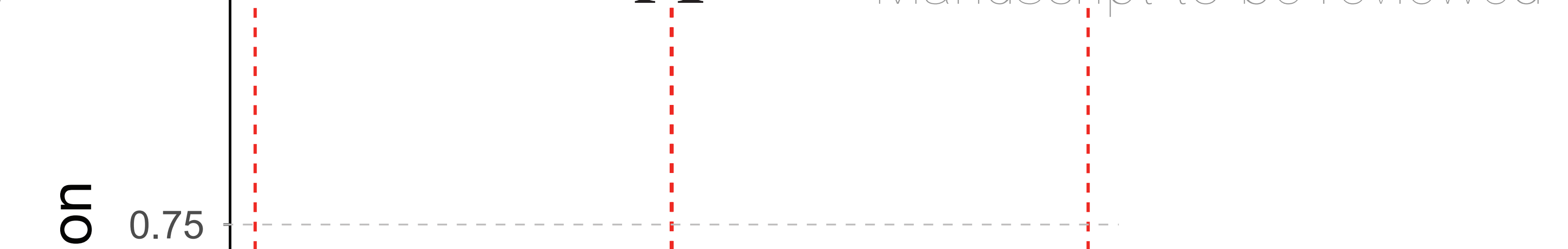

0.00

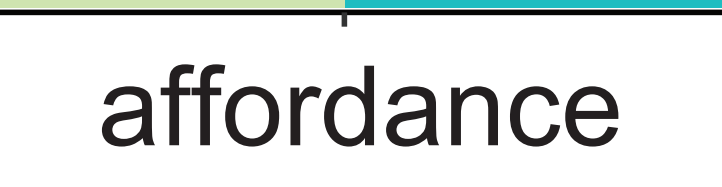

perceptual type of error

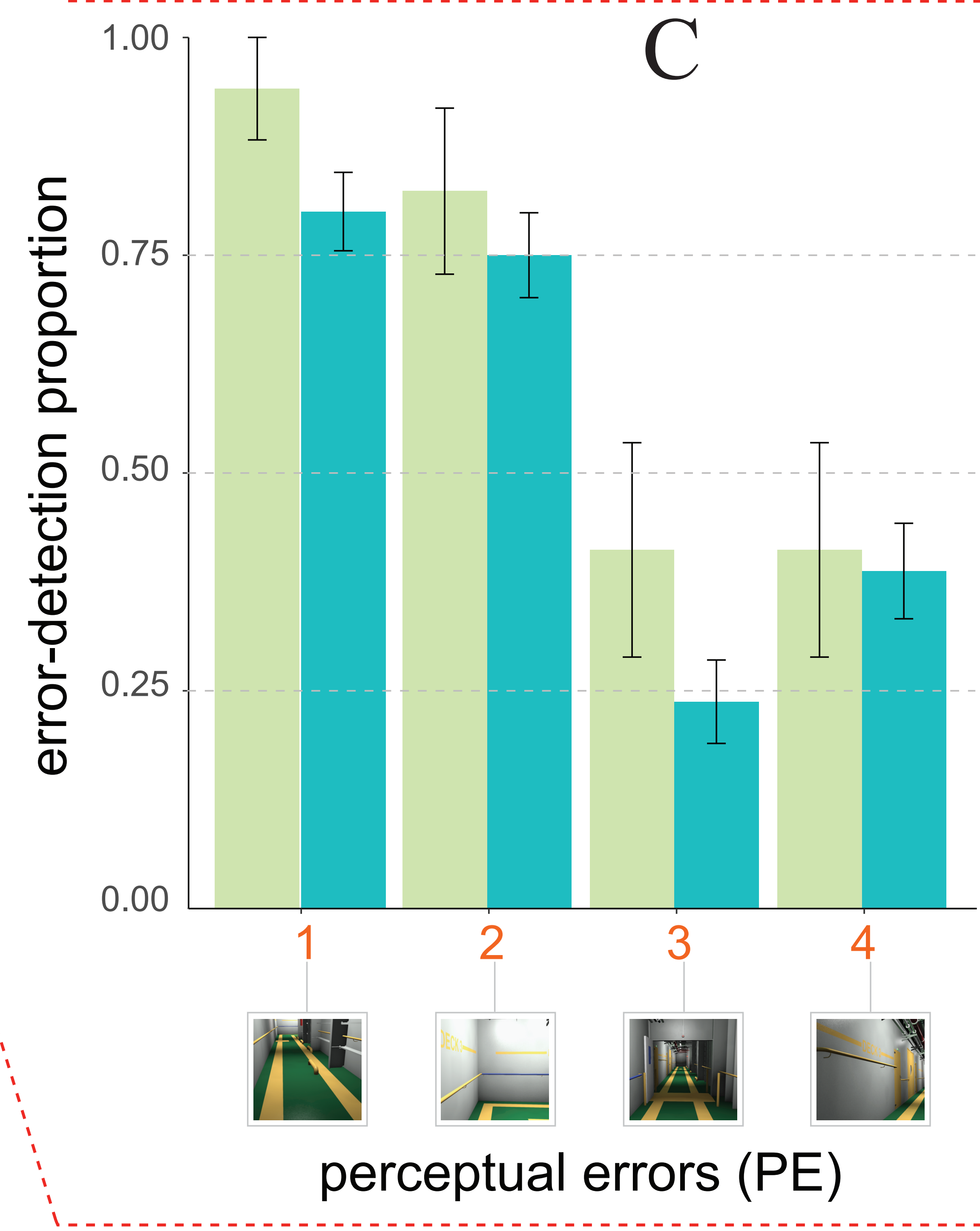

